\title{
Flow cytometry: basic principles and applications
}

\author{
Aysun Adan ${ }^{1}$, Günel Alizada ${ }^{2}$, Yağmur Kiraz ${ }^{1,2}$, Yusuf Baran ${ }^{1,2}$, and Ayten Nalbant ${ }^{2}$ \\ ${ }^{1}$ Faculty of Life and Natural Sciences, Abdullah Gül University, Kayseri, Turkey and ${ }^{2}$ Department of Molecular Biology and Genetics, İzmir Institute of \\ Technology, İzmir, Turkey
}

\begin{abstract}
Flow cytometry is a sophisticated instrument measuring multiple physical characteristics of a single cell such as size and granularity simultaneously as the cell flows in suspension through a measuring device. Its working depends on the light scattering features of the cells under investigation, which may be derived from dyes or monoclonal antibodies targeting either extracellular molecules located on the surface or intracellular molecules inside the cell. This approach makes flow cytometry a powerful tool for detailed analysis of complex populations in a short period of time. This review covers the general principles and selected applications of flow cytometry such as immunophenotyping of peripheral blood cells, analysis of apoptosis and detection of cytokines. Additionally, this report provides a basic understanding of flow cytometry technology essential for all users as well as the methods used to analyze and interpret the data. Moreover, recent progresses in flow cytometry have been discussed in order to give an opinion about the future importance of this technology.
\end{abstract}

\author{
Keywords \\ Apoptosis, cytokines, flow cytometer, \\ fluorescence, fluorescent-activated cell \\ sorting, histogram, immunophenotyping, \\ light scatter
}

\section{History}

Received 18 March 2015

Revised 12 October 2015

Accepted 13 October 2015

Published online 8 January 2016

\section{Introduction}

Historically, the first developed flow cytometry was a singleparameter instrument detecting only the size of cells. Currently, highly sophisticated instruments have evolved with the capability of detecting 14 parameters simultaneously (Wilkerson, 2012). Flow cytometry has the ability to measure the optical and fluorescence characteristics of a single cell or any other particle such as microorganisms, nuclei and chromosome preparations in a fluid stream when they pass through a light source (Macey, 2010). Size, granularity and fluorescent features of the cells, derived from either antibodies or dyes, are also examples of parameters used to analyze and differentiate the cells (Wilkerson, 2012). The underlying principle of flow cytometry is related to light scattering and fluorescence emission, which occurs as light from the excitation source (commonly a laser beam) that strikes the moving particles (Figure 1). The data obtained could give valuable information about biochemical, biophysical and molecular aspects of particles. Light scattering is directly related to structural and morphological properties of the cell while fluorescence emission derived from a

\footnotetext{
*These authors equally contributed to this work.

Address for correspondence: Assist. Prof. Dr. Ayten Nalbant, Molecular Immunology and Gene Regulation Laboratory, Department of Molecular Biology and Genetics, Izmir Institute of Technology, Urla, Izmir 35430, Turkey. Tel: + 90232 7507317. Fax: + 90232 7507303. E-mail: aytennalbant@iyte.edu.tr

Prof. Dr. Yusuf Baran, Department of Molecular Biology and Genetics, İzmir Institute of Technology, İzmir 35430, Turkey. Tel: + 90232 7507315. Fax: + 90232 7507300. E-mail: ybaran@gmail.com
}

fluorescence probe is proportional to the amount of fluorescent probe bound to the cell or cellular component (Macey, 2010).

There are two different types of flow cytometry - named as non-sorting and sorting. Non-sorting type can perform light scattering and fluorescence emission while the sorting type has the ability to sort particles as well. Fluorescent activated cell sorters (FACS) are flow cytometers that have the capacity to sort fluorescent-labeled cells from a mixed cell population (Wilkerson, 2012).

The main components of flow cytometers and cell sorters are basically fluidics, optics (excitation and collection), an electronic network (detectors) and a computer. The fluidics is responsible for directing liquid containing particles to the focused light source. The excitation optic focuses the light source on the cells/particles while collection optics transmits the light scatter or fluorescent light of the particle to an electronic network. The electronic network detects the signal and converts the signals to a digital data that is proportional to light intensity and the computer is also required to analyze data (Shapiro, 2004; Wilkerson, 2012).

Flow cytometry is used in various applications based on the detection of the membrane, cytoplasmic and nuclear antigens. Additionally, whole cells and cellular components such as organelles, nuclei, DNA, RNA, chromosomes, cytokines, hormones and protein content can also be investigated by flow cytometry. Analysis of cell proliferation and cell cycle, measurements of calcium flux and membrane potentials are the commonly used examples of methods developed for flow cytometry (Wlodkowic et al., 2011a,2013). 


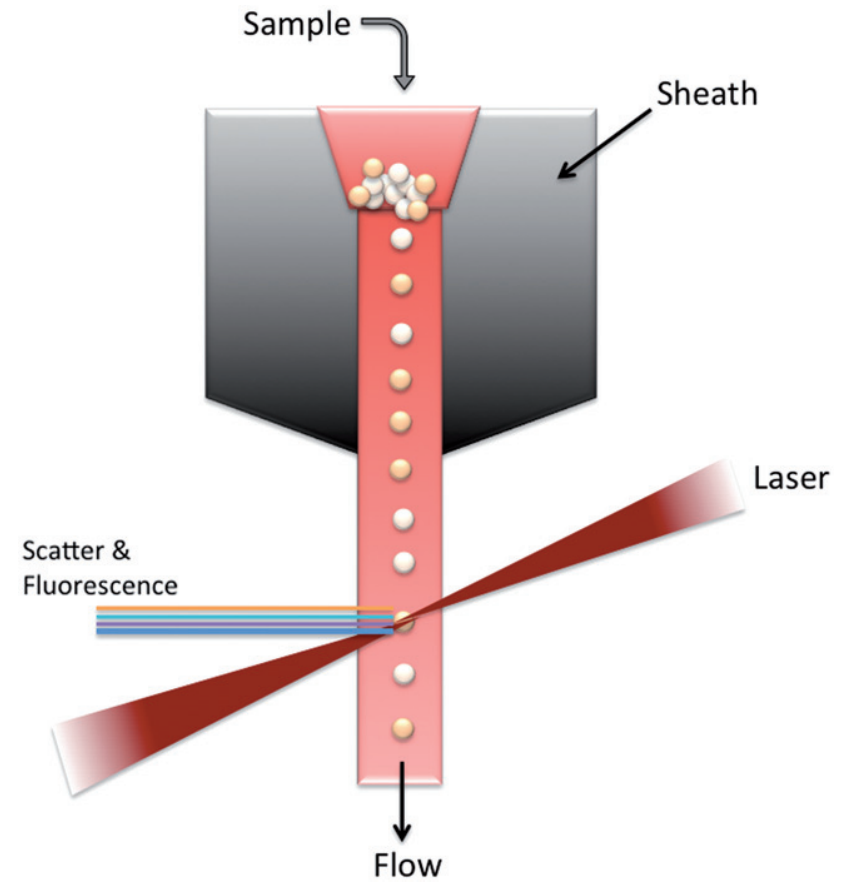

Figure 1. The underlying working principle of a flow cytometer.

Herein, we explained the general principles and selected applications of flow cytometry such as immunophenotyping of peripheral blood cells and analysis of apoptosis. Besides, we also discussed the basic data presentation and interpretation methods as a helpful material to the researchers who is interesting in flow cytometric analyses.

\section{Components of a flow cytometer}

\section{Fluidic system}

The fluidic system transports the cells from a solution through the instrument to obtain the data includes two components; sheath fluid and pressurized lines. Sheath fluid is a diluent (commonly phosphate-buffered saline (PBS)), which is injected into the flow chamber located at the heart of the instrument by pressurized lines. A pressurized airline also injects the suspended cells in the sample tube into the flow chamber. The sample stream becomes a central core in the sheath fluid stream called as a coaxial flow that is based on a pressure difference between the sheath fluid and sample stream (Macey, 2010; Wilkerson, 2012). The sample pressure is always greater than the sheath fluid pressure, making the cells align in a single file fashion through the laser beam. Therefore, this event allows uniform illumination of a cell called hydrodynamic focusing. The injection rate of the cells into the laser beam can be manipulated by the flow cytometer user based on the purpose of the analysis. For instance, high flow rates are common for qualitative measurements such as immunophenotyping of mammalian cells, whereas slow flow rates are appropriate for applications requiring higher resolution such as DNA content analysis. The slow flow rate makes the size of the sample stream smaller while it increases the uniformity and accuracy of the illumination (Macey, 2010; Shapiro, 2004; Wilkerson, 2012).

The critical parameter for providing proper interception between particles and the laser beam is the proper operation of fluidic components. Therefore, the operator must always ensure that the fluidics system is free of air bubbles and debris and is properly pressurized at all times.

\section{Optical system}

A flow cytometer has an optical bench that holds the excitation, which includes the laser and lenses and collection optics in fixed positions. The lenses are used to shape and focus the laser beam. Meanwhile, the laser produces light by energizing electrons to high energy orbitals with high voltage electricity. Photons of light are produced when these energized electrons fall back into their lower energy orbitals (Wilkerson, 2012). Light is deflected around the edges of the cell after the laser strikes the cells, also called as light scattering. Two types of light scatter occur named as forward scatter (FSC) and side scatters (SSC) (Figure 2). The factors affecting total light scatter include the membrane, nucleus, granularity of the cell, cell shape and surface topography. Generally, the size of a cell or a particle and its internal complexity specify the type of scatter. FSC light is a result of diffraction collected along the same axis as the laser beam. FCS is proportional to cell-surface area or size and suitable for detecting particles greater than a given size that makes it the most commonly used method for immunophenotyping. On the other hand, SSC light is a measurement of mostly refracted and reflected light, which is collected at approximately 90 degrees to the laser beam. SSC is proportional to cell granularity or internal complexity as important as the fluorescent light derived from fluorescent-labeled antibodies or dyes such as propidium iodide (PI) are reflected at the same angle as SSC. In order to differentiate the cell types in a heterogeneous population correlated measurements of FCS and SSC can be used (Reggeti \& Bienzle, 2011).

There is a variety of laser configurations in flow cytometers based on the type of fluorochromes being excited. The argon laser (a common laser with an excitation wavelength of $488 \mathrm{~nm}$ ) is used to excite many synthetic dyes such as fluorescein isothiocyanate (FITC) and natural fluorochrome dyes including algae and phytoplanktons, resulting in the emission of light at a higher wavelength. Many flow cytometers and sorters have additional lasers including ultraviolet that excite UV $(300-400 \mathrm{~nm})$ sensitive fluorochromes or the red diode which excites fluorochromes of the far red $(630 \mathrm{~nm})$ range (Macey, 2010; Wilkerson, 2012).

Collection optics consists of a collection of the lens to collect light emitted from the particle-laser beam interaction and a system of optical mirrors and filters to separate and then direct specified wavelengths of the collected light to the appropriate optical detectors (Reggeti \& Bienzle, 2011).

The specificity of a detector for a particular fluorescent dye is determined by placing a suitable filter that can be long pass, short pass and band pass filters. Band pass filters allow only a narrow range of wavelengths, which is close to the emission peak of the fluorescent dye to reach the detector. Short pass filters transmit wavelengths of light equal to or shorter than a specified wavelength whereas long pass filters transmit wavelengths of light equal to or longer than a specified wavelength (Reggeti \& Bienzle, 2011). 
Figure 2. Light scattering. FSC is proportional to size while SSC is proportional to cell granularity or internal complexity.

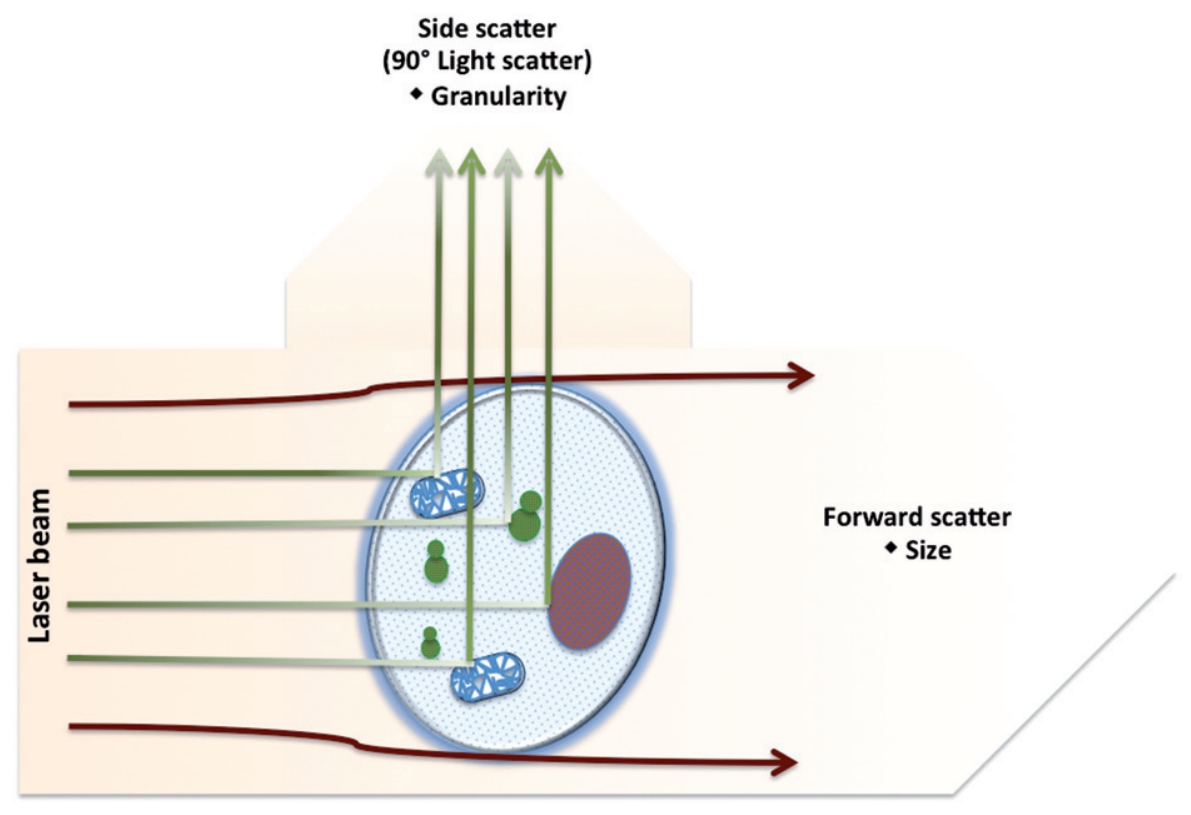

\section{Signal detection and processing}

Light signals generated as particles passing through the laser beam in a fluid stream are converted to voltages by photodetectors. Two types of detectors, photodiodes (PDs) and photomultiplier tubes (PMTs), can be preferred based on their sensitivity (Reggeti \& Bienzle, 2011; Snow, 2004). Additionally, the photocathodes of PMT have the greater sensitivity as compared to PD although they are known to convert light into photoelectrons in a more efficient manner. Therefore, PDs detect the stronger light signals generated by FSC, while PMTs are commonly used to detect the weaker signals generated by SSC and fluorescence (Figure 3) (Reggeti \& Bienzle, 2011).

The light signals captured by PMT or the PD are converted into a proportional number of electrons to create an electrical current. The electrical current travels to the amplifier and is converted to a voltage pulse. The maximum amount of scatter or fluorescence is obtained as the particle is in the center of the beam. When the particle leaves the beam, the pulse comes back down to the baseline (Snow, 2004).

Analog signal measured as an analog quantity is generated after the conversion of the initial light signal into an electrical current by the detectors. After that, this electrical current is amplified by two types of amplifier, which are linear and logarithmic. The obtained analog signal must be converted into digital signal by an analog to digital converters for computer processing. Then, the signals become a digital data that can be displayed as plots or histograms. Although the data obtained by using the two mentioned amplifiers is exactly the same, the distributions look different based on the approach used to convert an analog signal into digital data. Moreover, dynamic range is another important parameter to choose either linear or logarithmic amplification. Linear amplification is used if a limited dynamic range is required like in DNA analysis (a twofold difference in DNA content). On the other hand, logarithmic amplification is used if a much broader dynamic range is needed such as analysis of surface marker expression (100-10 000 fold) (Snow, 2004).

\section{Electrostatic cell sorting}

Electrostatic cell sorting is responsible for capturing and separation of the cells with predefined features. Once the cells of interest are collected, they can be used for further analysis such as microscopic, biochemical and functional studies. A single parameter or combination of several parameters can be used for cell sorting. To achieve cell sorting, cell sorters are commonly used. The general principle of flow cell sorters is based on the electrostatic deflection of charged droplets, some of which contain cells. In this method, the cells are injected through a nozzle to form a stream of regular droplets by applying a vibration to the nozzle. Then, these droplets pass through one or more laser beams and are charged by a charging electrode at the same time. Droplets can be deflected from the mainstream based on their given charges. Positively charged droplets are deflected toward a platinum plate of negative charge, negatively charged droplets are deflected toward the positively charged platinum plate and uncharged droplets are collected into a waste container (Figure 4) (Davies, 2010).

FACS is a specialized type of flow cytometry providing a method for sorting a heterogeneous mixture of fluorescenttagged cells into two or more containers, one cell at a time, based on the specific fluorescent characteristics of each cell (Davies, 2010). In this case, the operator selects the fluorescent cell of interest using a computer as they are passing through the laser beam. The tagged cells in the drop will be charged, which will be used to separate the cells into different collection vessels (Wilkerson, 2012).

\section{Principles of fluorescence}

A fluorescent compound has a range of specific wavelengths at which it absorbs light energy. This absorption of light causes an electron to rise from a ground state to a higher energy level (excited state). The excited electron quickly goes back to its ground state while giving the excess energy as a photon of light. This transition of energy is called fluorescence (Figure 5) (Ormerod, 2009). 
Figure 3. Signal processing and detection. Components of a flow cytometer are also indicated.

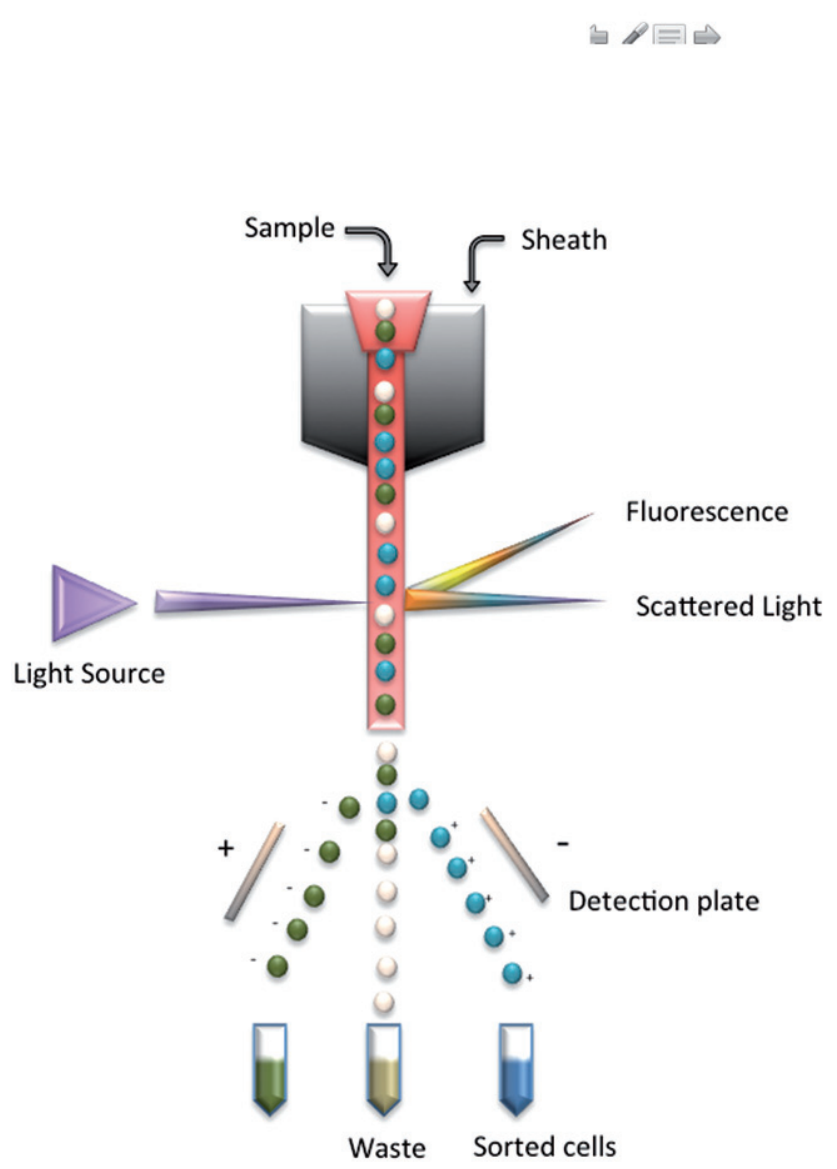

Figure 4. Sorting cells by droplet deflection.

\section{Fluorochromes and their properties}

The number of intrinsically fluorescent compounds in the cell is limited as well as its provided information. Therefore, the cells are usually stained with fluorescent probes called fluorochromes that are able to show the presence of
Fluidics system

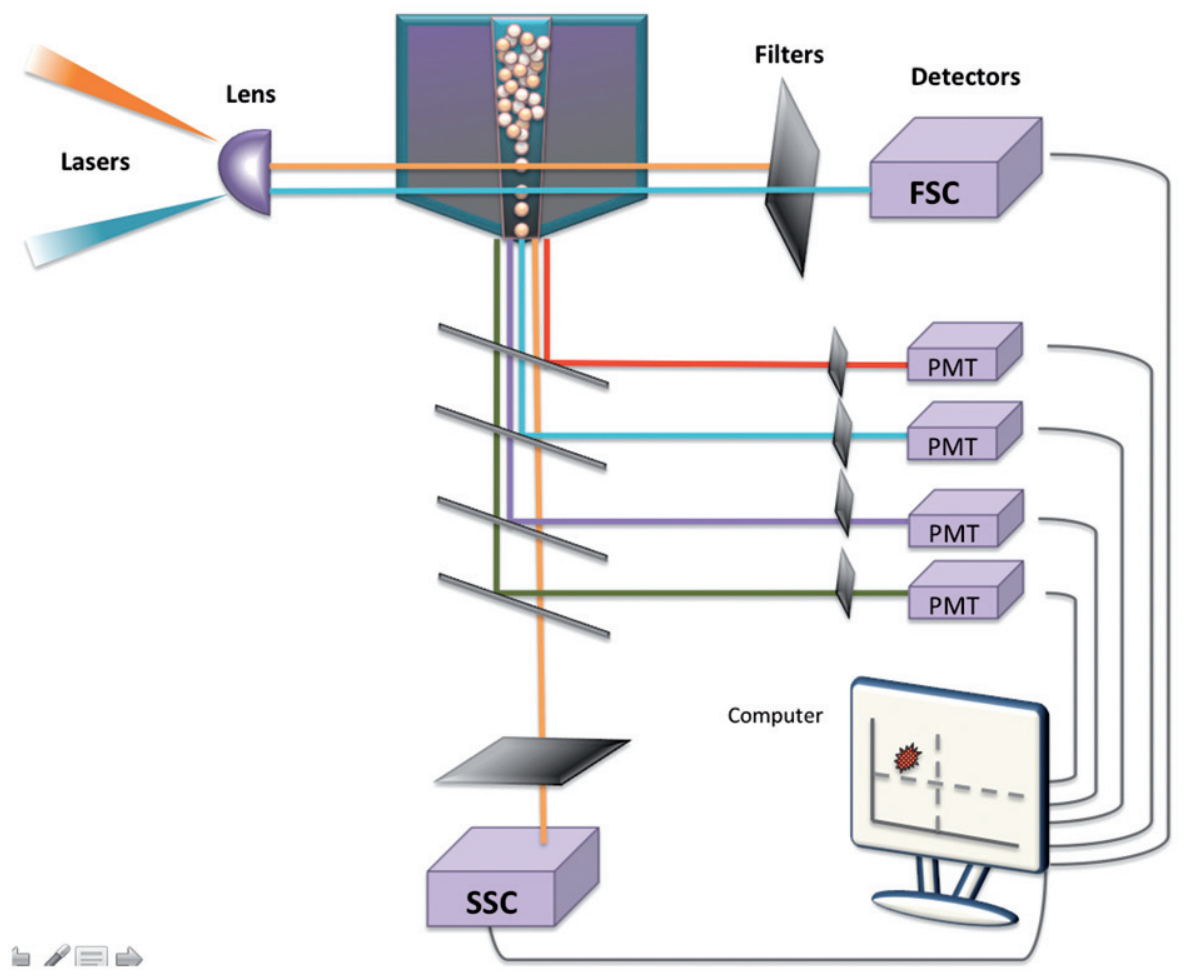

components that otherwise would not be visible. Fluorescent probes are used in a wide range of applications such as identification of different cell populations, cell surface receptors or intracellular organelles, cell sorting, immunophenotyping, determining nucleic acid content, measuring enzyme activity and apoptotic cell populations (Macey, 2010).

The important features of a fluorochrome include an absorption spectrum at which a fluorescent compound can be excited and a range of emitted wavelengths called its emission spectrum. The emission wavelength of any fluorochrome will always be longer than its excitation wavelength (Ormerod, 2009). The difference between the maxima in the wavelengths of absorption and emission is known as the Stoke's shift that determines how good a fluorochrome. The higher the Stoke's shift means, the greater the separation between the exciting and the emitted light (Figure 6) (Ormerod, 2009). Since the color of the exciting and emitting light is different, they can be separated from one another by using optical filters. The total photons of light being absorbed by the fluorochrome are related to the wavelength of excitation. For example, FITC absorbs the light within the range of $400-550 \mathrm{~nm}$, however, it gives maximum absorbance near $490 \mathrm{~nm}$ at which more photons are absorbed. Therefore, the fluorescence emission will be more intense. These optimal conditions are termed maximal absorbance and maximal emission wavelengths (Ormerod, 2009). In fluorescence detection, a positive signal is observed against a negative background, which makes this technique very sensitive. In this technique, multiparametric analysis of the cells is quite possible due to the detection of up to 14 compound fluorescing at different wavelengths. The majority of applications provide the usage of up to four fluorochromes (Ormerod, 2009). 
Figure 5. Fluorescence generation.

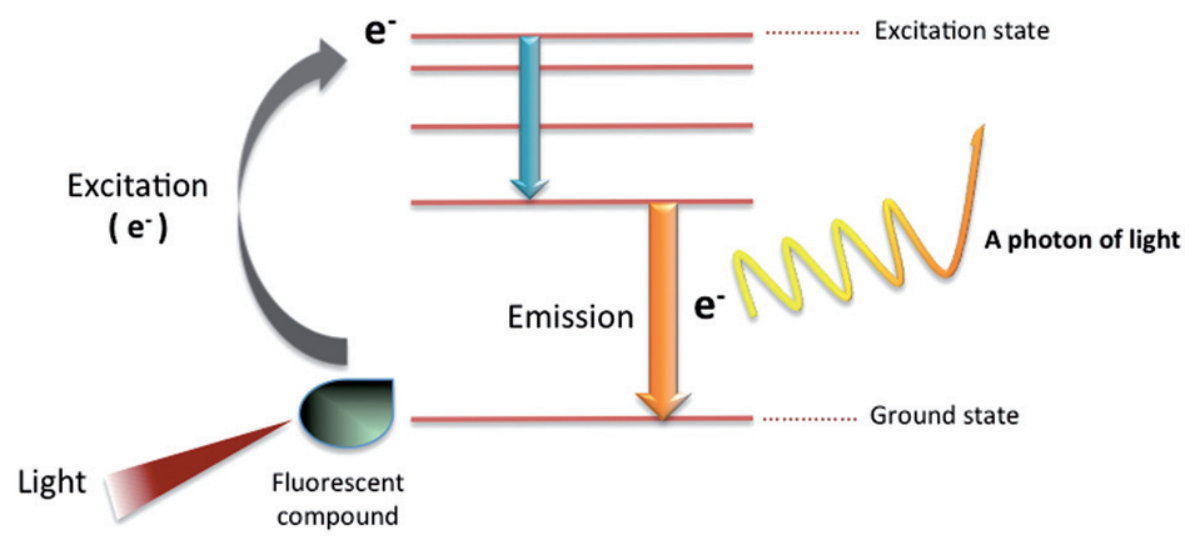

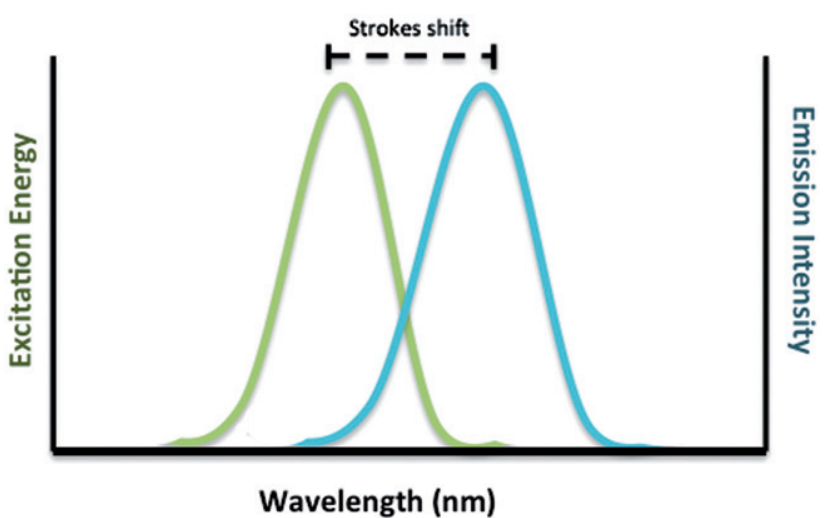

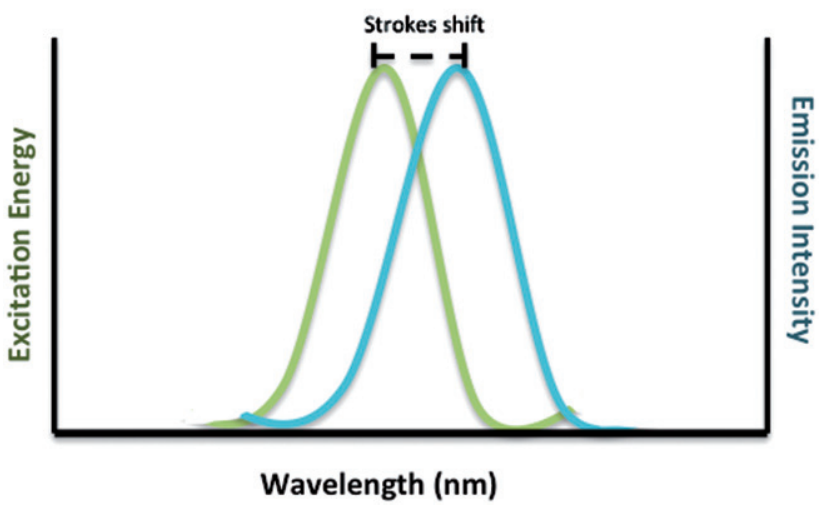

Figure 6. The absorption (green line) and emission (blue line) spectra of a fluorochrome. The difference between the peak wavelengths of the absorption and emission spectra is known as the Stokes shift. The higher the Stoke's shift means the greater the separation between the exciting and the emitted light (top).

\section{Fluorochromes used in flow cytometry}

Fluorochromes used in flow cytometry are classified into several groups including fluorochromes used to label proteins covalently, fluorochromes for nucleic acids and reporter molecules.

In order to label proteins covalently, the probe is commonly selected as an antibody. However, other proteins such as a lectin, hormone, avidin or streptavidin, or even c-DNA may be labeled using various fluorochromes. The most widely used fluorochromes for labeling antibodies include FITC, phycoerythrin (PE) and allophycocyanin (APC) (Ormerod, 2009). The selection of the most suitable fluorochrome is an important issue and depends on the laser to be used. If an argon-ion laser including flow cytometer is going to be used, the first choice will be FITC, since there are numerous FITC-labeled antibodies available. PE is the second choice of color for a similar reason.

FITC easily reacts with the amino groups on the lysine residues in the protein and produces moderately stable conjugates. FITC (excitation/emission maxima approx 495/ $520 \mathrm{~nm}$ ) is a good fluorochrome for single-color staining since its maximum absorbance near $490 \mathrm{~nm}$. On the other hand, its emission with longer wavelengths makes it inappropriate for multicolor applications. Moreover, its fluorescence is highly $\mathrm{pH}$-sensitive and subjected to photobleaching with a high rate. To solve these problems, various FITC derivatives have been developed such as Alexa ${ }^{\mathrm{TM}}$ series with greater photostability and increased fluorescence (Macey, 2010).

PE (excitation/emission maxima $495565 / 578 \mathrm{~nm}$ ) and APC (excitation/emission maxima $650 / 660 \mathrm{~nm}$ ) are called phycobiliproteins that are the components of photosynthetic systems. They have good light absorption and high fluorescence intensities. Although their fluorescence is about 30-fold greater than that of fluorescein, in practice, cells labeled with phycobiliprotein antibodies have fluorescence intensities between five- and 10-fold greater than those labeled with FITC-labeled antibody. Although using an argon laser excites FITC and PE, the excitation of APC needs helium-neon laser due to its higher $(650 \mathrm{~nm})$ absorption maxima. The major drawback of using phycobiliproteins is related to their higher molecular weight, causing steric changes when conjugated to proteins. They can also give higher backgrounds if the cells are not washed properly (Telford, 2015).

The development of tandem dyes, containing two fluorochromes, has increased the number of labeled proteins to be used. Examples include conjugates of PE and APC with various cyanine dyes, for instance, $\mathrm{PE}-\mathrm{Cy}^{\mathrm{TM}} 5$ and $\mathrm{APC}-\mathrm{Cy} 7$ (Hulspas et al., 2009). In tandem dyes, when the first dye is excited and reaches its maximal absorbance, it transfers all its energy to the second dye located in close proximity. As a result, this second fluorochrome is activated and produces the fluorescence emission. This process is called fluorescence resonance energy transfer (FRET). It is a good way to obtain higher stokes shifts that increase the number of colors analyzed from a single laser wavelength (Leavesley et al., 2013). 
Fluorochromes used to stain nucleic acids in flow cytometry have some main applications including DNA quantification, the evaluation of membrane integrity and measurement of ploidy and cell cycle analysis. At present, there are many dyes that can stain both RNA and DNA such as ethidium bromide (EB) and PI. It is important to remove RNA with RNase when quantifying DNA. EB and PI intercalate between the bases in double-stranded nucleic acids. PI is excited by blue light and gives red fluorescence. PI can be used in combination with FITC for simultaneous measurement of antibody binding and DNA content if an argon laser is tuned to $488 \mathrm{~nm}$. 7-Aminoactinomycin D (7-ADD) characterized by its preferential binding to GC-rich regions of DNA is generally removed by live cells and used to stain DNA of dead cells. It can be excited by an argon laser and used with FITC- and PE-labeled antibodies. There are also UV-excited dyes such as Hoechst and DAPI that bind to AT-rich regions of DNA. Although they are membrane permeable dyes, dead cells can uptake them more easily (Telford et al., 2011; Wlodkowic et al., 2011b).

Reporter molecules are also used in flow cytometric analysis. For instance, green fluorescent protein (GFP) is excited at $488 \mathrm{~nm}$ and fluorescent green. GFP and its mutant forms are used in various applications such sorting of GFP transfected cells (Telford et al., 2012). Another reporter molecule is JC-1 dye used to measure mitochondrial membrane potential. JC-1 dye accumulates in mitochondria of healthy cells by forming red JC-1 aggregates with emission at $590 \mathrm{~nm}$ (Wlodkowic et al., 2011b). In addition, there are several other reporter molecules to detect several changes in experimental cells such as production of the reactive oxygen species, measurement of intracellular calcium ions and changes in intracellular pH (Macey, 2010).

\section{Fluorescence compensation}

Each fluorochrome has a wide emission spectrum resulting in some overlap between the fluorochromes when multiple fluorochromes are used. This situation is called spectral overlap and being exemplified as the emission spectra for FITC and PE are overlapped at the same wavelengths at which some of the light emitted by fluorescein will be pass through the filter used for PE. This spectral overlap is corrected by subtracting a fraction of the FITC signal from the PE signal or vice versa. This process is named as color compensation, that also calculates how much interference (as a percent) a fluorochrome will have a channel that was not assigned specifically to measure it (Roederer, 2001). In order to determine how much compensation should be applied to the dataset, some control readings must be performed first. One of the fluorochromes should be read on itself and then the percent of its detectable total emission in the channel specific for second fluorochrome is determined. This procedure should be repeated for a second fluorochrome as well (Figure 7) (Ormerod, 2009).

\section{Data analysis}

Flow cytometry data analysis has been considered as the most critical parameter for biological experiments. The major principles of data analysis are to selectively show the cells of interest and to find out more about your cells. This method is called "gating" in flow cytometry as well as a gate can be defined as one or more regions combined. A region can be defined as a set of points carefully selected by the user that determines an area on a graph. Several regions can be defined on the same graph. These parameters can provide you with an opportunity to eliminate results from the unwanted particles such as dead cells and debris. The most common application of gating strategy is to use FSC and SSC plots (Figure 8). The different physical characteristics of white blood cells, granulocytes, monocytes and lymphocytes, allow them to be distinguished from each other.

\section{Gates and regions}

A subset of data can be defined by gating methods. Additionally, a gate is a numerical or graphical boundary that can be used to define the characteristics of particles for further analysis. For example, in a blood sample containing a mixed population of cells, you might want to restrict your analysis to only the lymphocytes. Based on FSC or cell size, a gate can be set on the FSC versus SSC plot for the analysis of lymphocytes. The resulting display would reflect the fluorescence properties of only the lymphocytes (Figure 9). The data can be displayed by several different plot types. These range from histograms to 2-D plots such as dot plots, contour and density plots, to 3-D plots such as a tomogram plot.

\section{Single parameter analysis}

A histogram is a single parameter plot where the $\mathrm{x}$-axis represents the parameter's signal value in channel numbers and the y-axis represents the number of events per channel number. Each event placed in the channel corresponds to its signal value. Signals with identical intensities accumulate in the same channel. Brighter signals are displayed in channels to the right of the dimmer signals. Ideally, flow cytometry will produce a single distinct peak (Figure 10). However, flow analysis is sometimes marked on a mixed population of cells resulting in several peaks on the histogram.

\section{Two parameter analysis}

Alternate methods of data display are available for twoparameter plots. These are graphs that display two measurement parameters, one on the $\mathrm{x}$-axis and one on the $\mathrm{y}$-axis and the cell count as a density (dot) plot or contour map. A dot plot provides a two-parameter display of data and each dot represents one or more events (particles) while density plot displays two parameters as a frequency distribution (Figure 11). The color is used to code the different frequencies of events in density plot (Figure 12). The contour plot is the same as a density plot, except the density of the cells is displayed as contour lines.

A quadrant marker divides two-parameter plots into four sections to discriminate populations as negative, single positive or double positive. The upper-right quadrant of the plot indicates cells positive for both fluorescent marker or double positive. The lower-left quadrant displays events that are negative for both markers. The upper-left quadrant of the 

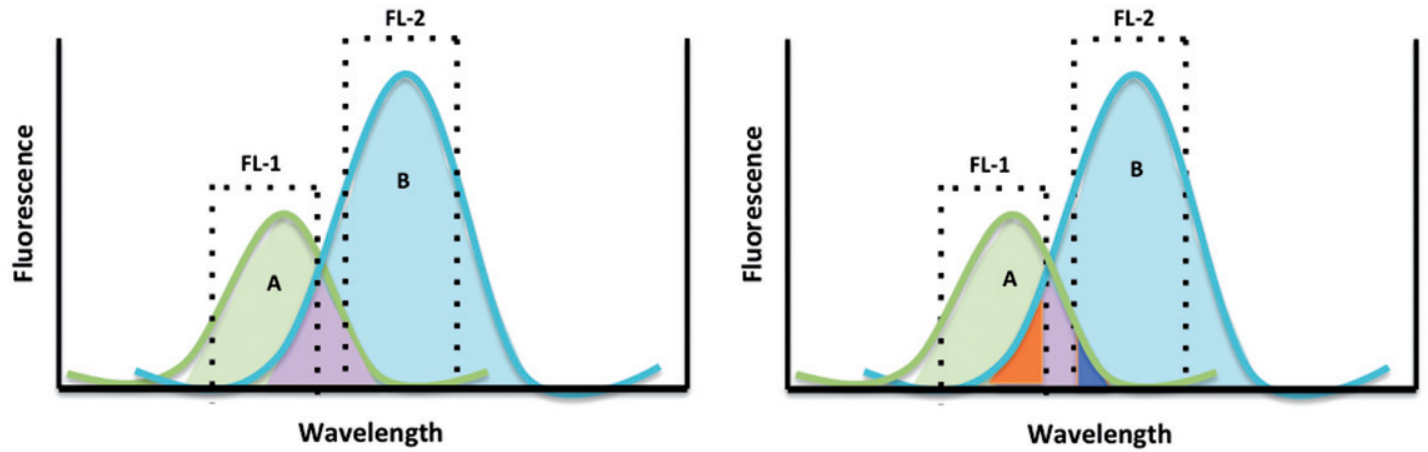

Figure 7. Explanation of fluorescence compensation by using two imaginary fluorochromes A and B. A is measured in the FL-1 channel while B is in FL-2 channel. Orange part indicates the proportion of B that overlaps into the FL-1 channel. Blue part indicates the proportion of A that enters into the FL-2 channel.

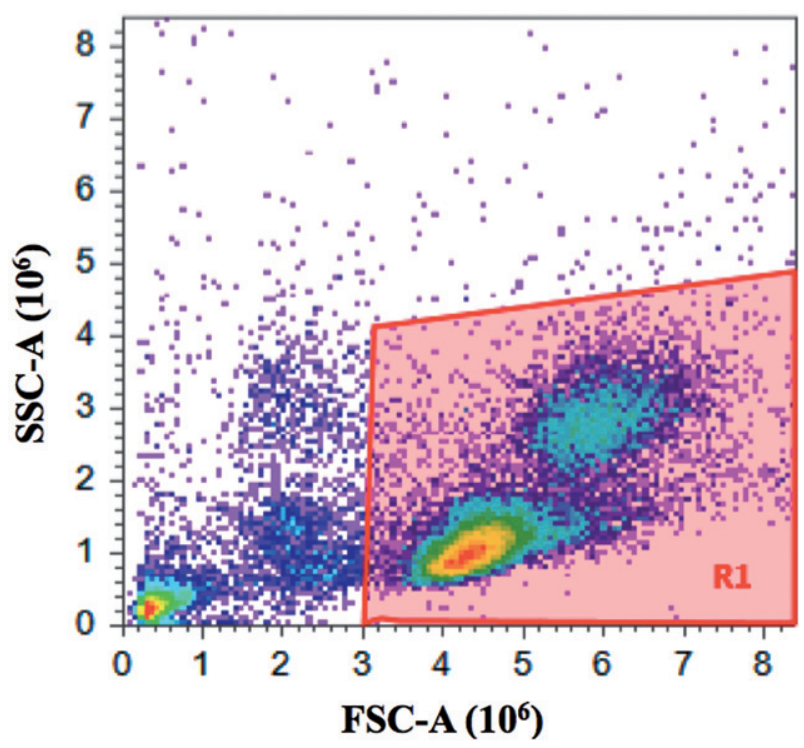

Figure 8. Analysis of freshly isolated human peripheral blood mononuclear (PBMCs) cells using SSC/FSC. Separation of PBMCs from whole blood is most commonly performed using Ficoll (Böyum, 1968). FSC correlates with the cell volume while SSC correlates with a granularity of the cell. Neutrophil, monocytes and lymphocyte populations were identified with their light scattering characteristics alone. A total of 30000 events were acquired for analysis.

plot indicates cells only positive for the y-axis parameter. The lower-right quadrant contains events that are positive for the $\mathrm{x}$-axis marker but negative for the $\mathrm{y}$-axis fluorescent marker (Figure 13).

\section{Common protocols}

Flow cytometry is a powerful technology to study different types of cells and their functions. The most common cell staining principles for identifying and investigating functions of a single cell are surface and intracellular staining are described in detailed below.

\section{Cell surface staining}

Cell surface antigens localized on the plasma membrane of the cells are the most common proteins to use for flow cytometry to identify and characterize the cell types. Plasma membrane proteins are easily accessible to the antibody for

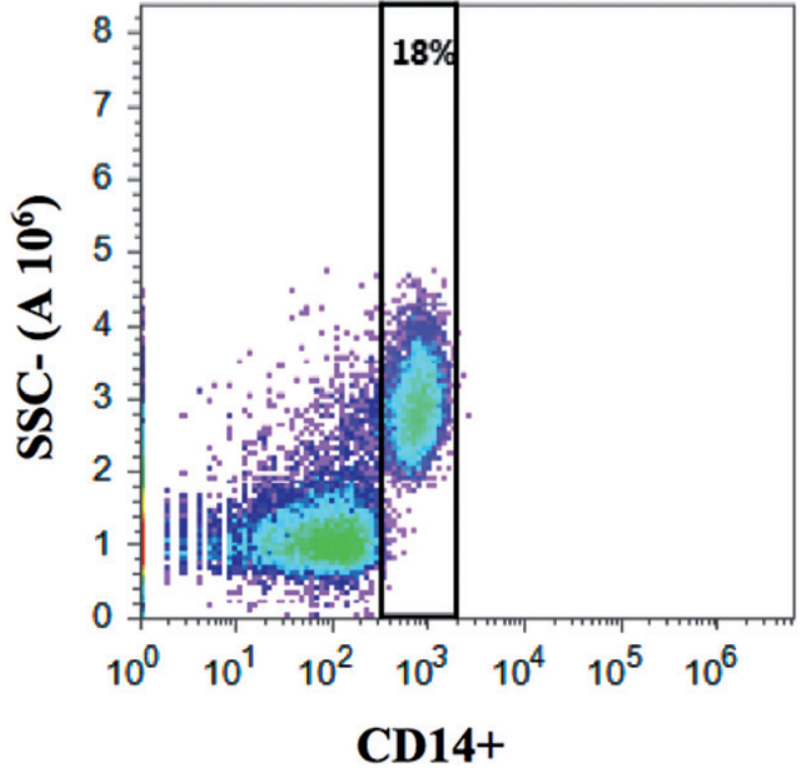

Figure 9. Rectangular gates. Peripheral blood mononuclear cells (PBMCs) are plotted as SSC on the y-axis versus CD14 fluorescence on the $\mathrm{X}$-axis. Freshly isolated human PBMCs were washed and labeled with CD14 monoclonal antibody (BioLegend, San Diego, CA) for cell surface antigen CD14. The labeled cells were analyzed by Attune flow cytometry. Rectangular gate indicates CD14 positive monocytes in PBMC population.

cell surface staining. To obtain the best result of staining, protocols should be optimized in terms of antibody concentration, incubation time and temperature for each flow cytometry experiment.

Cell surface antigens either on cultured or freshly isolated cells can also be stained. Freshly isolated peripheral blood mononuclear cells (PBMCs) were labeled with the selected combination of cell surface antibodies including anti-CD3, anti-CD4 and anti-CD14 (BD Biosciences, Franklin Lakes, $\mathrm{NJ}$ ) for $15 \mathrm{~min}$ at $4{ }^{\circ} \mathrm{C}$ in the dark. Then, cells were washed two times by centrifugation at $400 \mathrm{~g}$ for $5 \mathrm{~min}$ and resuspend in the ice-cold PBS. Then, cells were analyzed by flow cytometry (Figures 13 and 14). Attune Acoustic Flow Cytometer (Life Technologies, Carlsbad, CA) has been used to acquire all of the samples and data presented in this review were analyzed by Attune Cytometer Software (Life Technologies, Carlsbad, CA) version 2.1.0. 

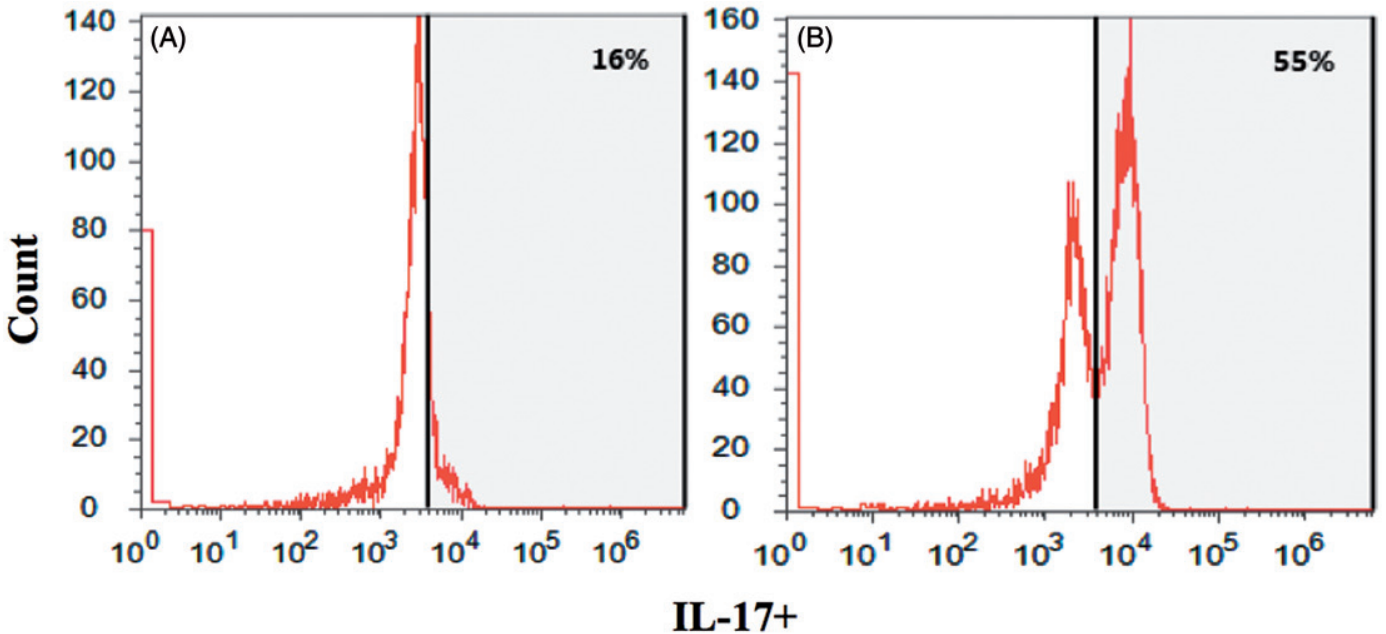

Figure 10. A single-parameter histogram. The x-axis represents the parameter's signal value in channel numbers (Count) and the y-axis represents the number of events per channel number (IL17 positive cells). Naïve CD4 + T cells were stimulated with Th17 culture condition during 7th d. The cells were fixed with Fixation Buffer and permeabilized with Permeabilization/Wash Buffer I (BioLegend). Both (A) unstimulated and (B) stimulated cells were stained with Anti-Human IL-17A Fluorescein-conjugated Antibody (BioLegend). Finally, cells were washed and analyzed with Attune flow cytometry.

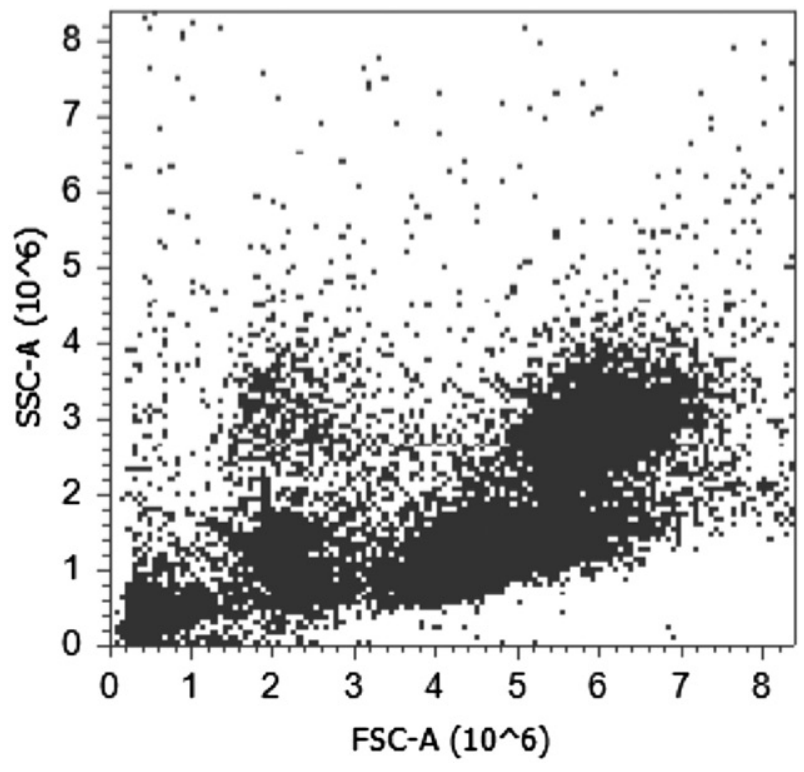

Figure 11. Dot plot of PBMC. Cell subpopulations based on FSC versus SSC were analyzed by flow cytometer. Correlated measurements of FSC and SSC can allow cell population to be distinguished from each other and from cellular contaminants. Dead cells have higher side scatter and lower forward scatter than living cells.

\section{Intracellular antigen staining}

Intracellular proteins of cells can also be detected by flow cytometry. To stain the intracellular proteins, cells are fixed with formaldehyde to stabilize the cell membrane antigens and then permeabilized with detergent or alcohol to allow antibodies to enter into the cell. It is important to focus on the location of proteins that will be stained. The presence of proteins either in cytosol or nucleus of the cell will dictate the protocol and buffer system that will be used to get optimum staining of a protein of interest. It is also possible that cell surface and intracellular staining protocols can be used

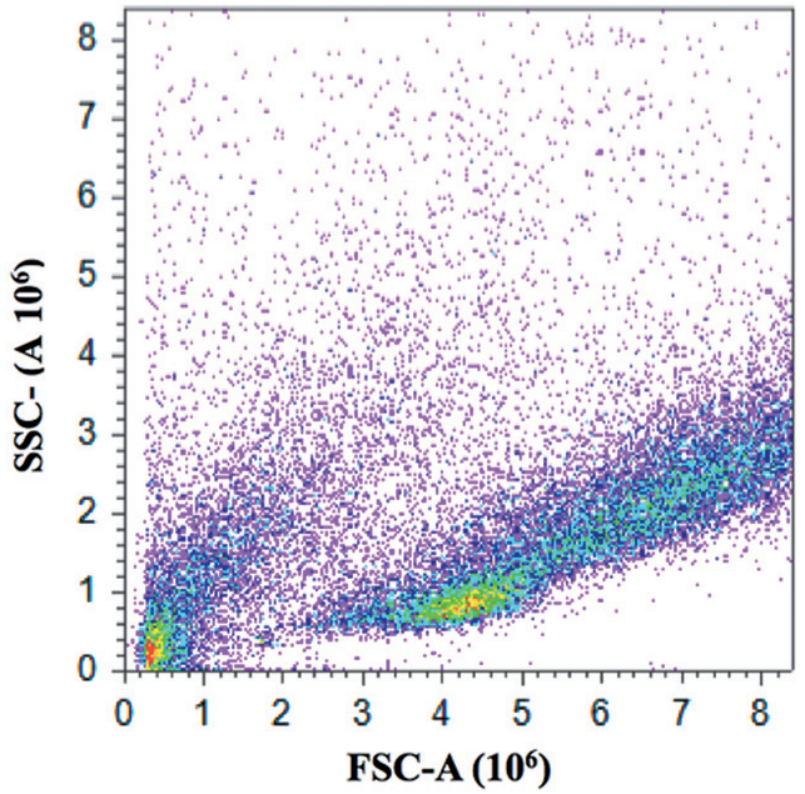

Figure 12. Density plots of a stimulated naive CD4 + T cells. Isolation of naive $\mathrm{CD}^{+} \mathrm{T}$ helper cells from PBMCs using the Naive $\mathrm{CD} 4^{+} \mathrm{T}$ Cell Isolation Kit II (Miltenyi Biotec, Bergisch Gladbach, Germany). Naive CD4 T cells were stimulated with Th17 polarizing conditions for $7 \mathrm{~d}$. It shows all the cells where the different colors represent the density of the cells at a given position.

simultaneously at the single-cell level by flow cytometry to gain more information about the specific cell.

Bcl-2 or active caspase- 3 staining protocols were described here as an example of intracellular protein staining. Cultured or freshly isolated cells were harvested, washed with cold PBS and fixed with Cytofix/Cytoperm solution (Pharmingen containing PBS with $4 \%$ paraformaldehyde and $0.1 \%$ saponin for $30 \mathrm{~min}$ on ice. Cells were then washed with wash buffer including PBS plus $0.1 \%$ sodium azide and $1.0 \%$ fetal bovine serum (FBS). Cells were re-suspended and permeabilized in permeabilization buffer containing PBS with $1 \%$ FBS, $0.1 \%$ 


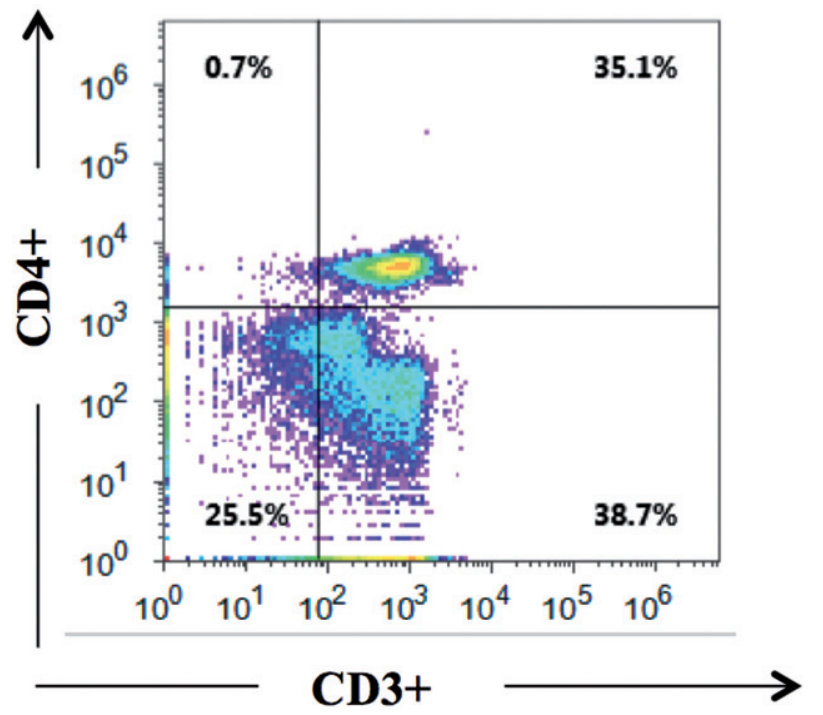

Figure 13. Density plot quadrants. Freshly isolated human peripheral blood mononuclear cells were labeled with CD4 and CD3 antibodies for cell surface antigens (BioLegend). The labeled cells were analyzed by Attune flow cytometry. Cells were gated on lymphocytes. The $\mathrm{x}$-axis represents $\mathrm{CD} 3$ positive and the $\mathrm{y}$-axis represents $\mathrm{CD} 4$ positive cells. Upper right quadrant shows CD3 and CD4 double positive cells.

sodium azide and $0.1 \%$ saponin for $10 \mathrm{~min}$ on ice. Then, cells were stained for $20 \mathrm{~min}$ at $4{ }^{\circ} \mathrm{C}$ in the dark with monoclonal antibodies specific for intracellular antigens such as Bcl-2 (Figure 15) or active caspase-3 (Figure 16). Following two times washing, cells were analyzed by flow cytometry.

\section{Applications of flow cytometry}

\section{Phenotypic characterization of blood cells}

Immunophenotyping or phenotypic characterization of cells is the identification and quantification of a specific cell group in the mixed population using flow cytometry e.g. immune cells of the blood. Specific cell surface membrane proteins, which are known as markers, can be detected by using antibodies. These specific antigens can be expressed on more than one cell type.

Therefore, immunophenotyping of the cells can be accomplished by staining of a single cell simultaneously with two or more antibodies. Phenotypic characterization of blood cells is seen in Figure 17. Human PBMCs were first isolated by Ficoll-Hypaque density gradient centrifugation (Böyum, 1968). Then, PBMCs were labeled with the selected combination of cell surface antibodies including anti-CD3, anti-CD4 and anti-CD14 (BD Biosciences) (Figure 14). Cell surface labeling was carried out as described in "Cell Surface Staining', section. Cell surface staining section and labeled cells were analyzed by flow cytometry.

\section{Measurement of apoptosis markers}

Rapid and quantitative measurement of apoptotic cells can be detected by flow cytometry. The methods for the detection of cells undergoing apoptosis (programmed cell death) should detect apoptotic properties of cells rapidly and allow them to keep their natural state in order to obtain the most reliable results. Many different flow cytometric methods for the assessment of apoptosis in cells have been described including measuring plasma membranes changes, detection of active caspase- 3 and DNA fragmentation.

\section{Cell viability}

The measurement of cell viability plays an important role in all kinds of cells. Viability analysis can provide information about the viable (live) and non-viable (dead) cells. Non-viable cells in your samples can non-specifically bind to your antibodies resulting in false positives and ultimately inaccurate results. The detection of cell viability by flow cytometry is based on a simple yet powerful principle (Wlodkowic et al., 2012). The viability of cells can easily be determined in flow cytometry by adding one of DNA binding dyes at a relatively low concentration to a population of cells. Discrimination between live and dead cells in flow cytometric analysis can be carried out based the usage of several different fluorochromes including 7-amino actinomycin D (7-AAD) (Figure 17), PI and DAPI.

\section{Detection of plasma membrane changes}

Apoptosis is characterized by a variety of molecular and morphological changes. One of the earliest events in an apoptotic cascade is the alteration in the plasma membrane. The membrane phosphatidylserine is translocated from the inner to the outer leaflet without affecting the plasma membrane integrity, which leads to detect the culmination of apoptosis by the loss of plasma membrane integrity (Vermes et al., 1995).

Cells were washed and labeled with Annexin V and 7-AAD in the presence of $\mathrm{Ca}^{++}$binding buffer (Becton Dickinson, Mountain View, CA) for $30 \mathrm{~min}$ and analyzed by flow cytometry within $1 \mathrm{~h}$ in order to measure the plasma membrane changes Annexin V-positive, 7-AAD-negative cells were defined as early apoptotic cells with an intact cell membrane with externalized phosphatidylserine (Figure 18). In contrast, Annexin V-positive and 7-AADpositive cells were defined as dead cells.

\section{Detection of active caspase-3 activity}

Another molecular hallmark of apoptosis is the activation of caspases. Caspases are inactive enzymes and must become active during apoptosis. One of the effector caspases activated at the late apoptosis stage is caspase-3. Active caspases-3 initiates series of events that ultimately kill a cell (Lessene et al., 2008; Wlodkowic et al., 2012). The active caspase-3 can be detected and analyzed with flow cytometry (Figure 16).

\section{Detection of mitochondrial proteins}

$\mathrm{Bcl} 2$ is a human proto-oncogene located in the membranes of the endoplasmic reticulum, nuclear envelope and in the outer membrane of mitochondria. Preclinical studies demonstrated that $\mathrm{Bcl} 2$ is anti-apoptotic intracellular protein and the decreased $\mathrm{Bcl} 2$ level indicates progression of apoptosis (Wlodkowic et al., 2012). In order to detect Bcl2 activity, cells were fixed and permeabilized. Then fluorescenceconjugated $\mathrm{Bcl} 2$ antibody was added into cells and analyzed by flow cytometry (Figure 15). 
(A)

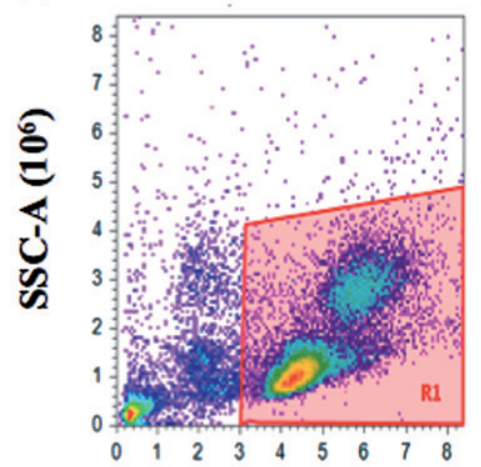

FSC-A

(E)

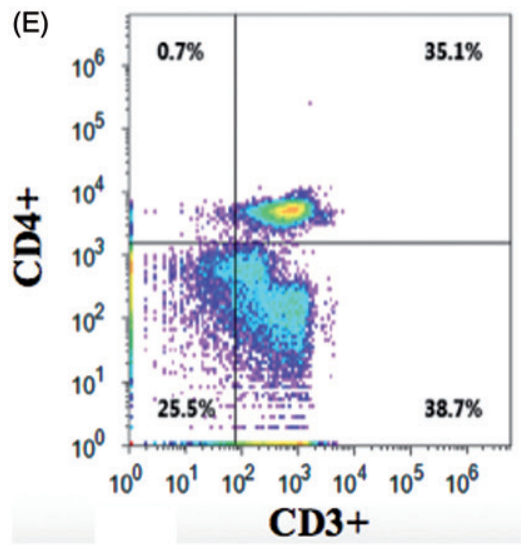

(B)

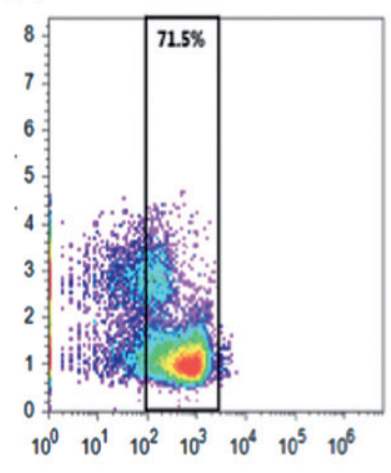

CD3+
(C)

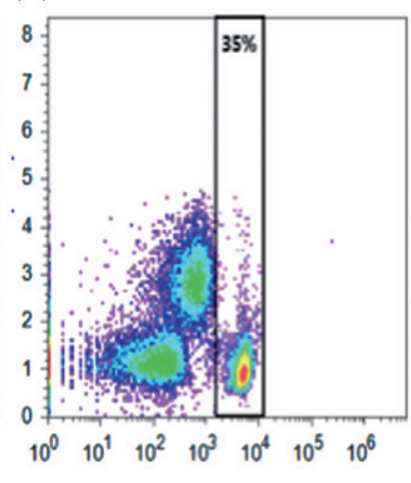

CD4+
(D)

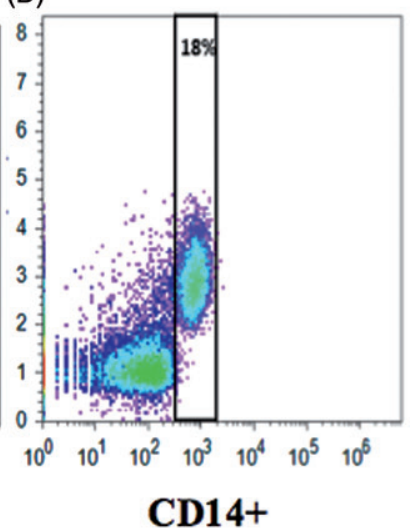

Figure 14. (A) Phenotypic characterization of PBMCs. Human peripheral blood mononuclear cells were gated using FSC/SSC parameters. PBMC were labeled with monoclonal antibodies for CD4, CD3 and CD14 cell surface markers (BioLegend). The labeled cells were analyzed by Attune flow cytometry. Cells were gated on lymphocytes. (B-D) Rectangular gates show CD3, CD4 and CD14 positive cells. (E-G) Two-parameter dot plots quadrants show (from left to right) CD4 and CD3 double positive cells and CD14 positive cells only.

Figure 15. The expression of Bcl-2 in CD4 T cells. Naïve CD4 T cells were cultured using Th17 culture condition during $9 \mathrm{~d}$. Golgi Stop was added to the cultures before $4 \mathrm{~h}$ for intracellular immunofluorescence staining. Cells were washed and cell surface was stained with CD4 antibodies (BioLegend). After fixation and permeabilization with Fixation and Permeabilization Buffer Set (BioLegend), fluorescence conjugating with the $\mathrm{Bcl} 2$ antibody (BioLegend) was added into cells. Bcl-2 and CD4 expression was analyzed by double immunostaining by Attune flow cytometry, and populations were all CD4 gated. (A) Rectangular gate indicates $\mathrm{Bcl} 2$ positive in $\mathrm{CD} 4+\mathrm{T}$ cell population and (B) right quadrant shows Bcl2 and CD4 double positive cells.
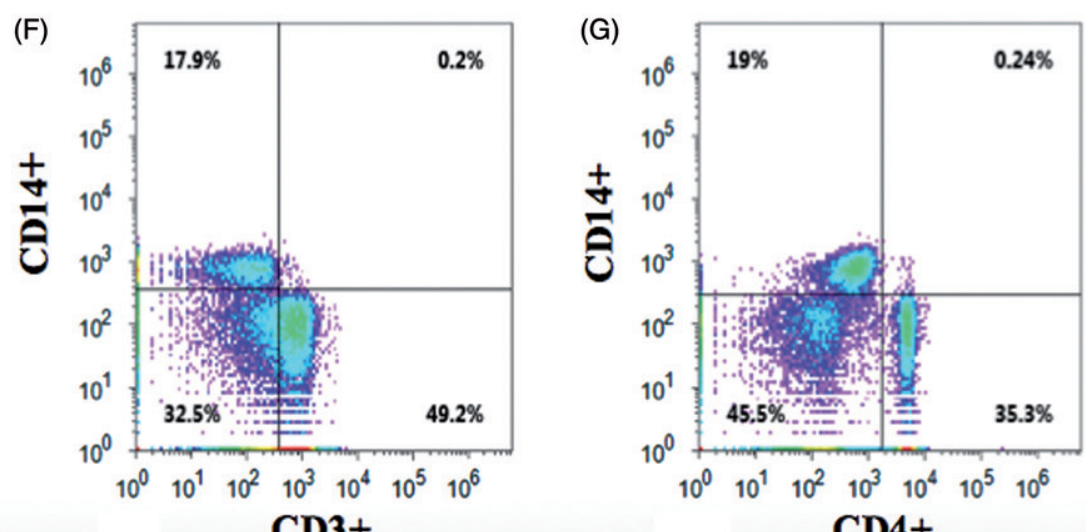
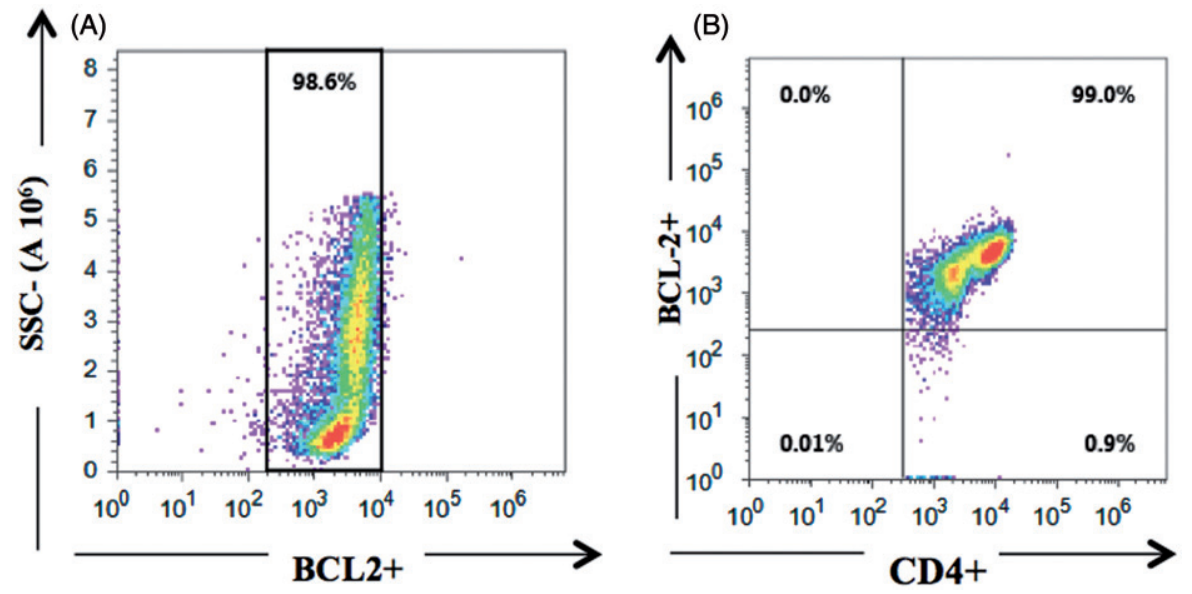

\section{DNA fragmentation}

Programmed cell death is accompanied by degradation of chromosomal DNA. Technically, the easiest characteristic to detect is a loss of DNA from permeabilized cells due to DNA fragmentation (Wlodkowic et al., 2009). After permeabilization, the fragmented $182 \mathrm{bp}$ DNA multimers leak out of the cell. Providing that, the cells are stained with PI, then a DNA profile representing cells in G1, S- and G2/M phases will be observed together with apoptotic cells being represented by a sub G0/G1 population seen to the left of the $\mathrm{G} 0 / \mathrm{G} 1$ peak.

The advantage of this method that it is very fast, inexpensive and simple. Furthermore, it detects cumulative apoptosis and is applicable to most cell types. Moreover, the method may not detect cells induced to apoptosis in the G2/M phase.

An alternative method to identify DNA fragmentation involves the identification of the nicks (or strand breaks) using the TUNNEL technique. This protocol is used for detection and 
Figure 16. The detection of active-caspase-3. Human naïve CD4 T cells were cultured in Th17 polarizing conditions. (A) IMDM was used as a negative control. The expression of active caspase- 3 was measured at 7 th $d$. In order to detect the active caspase- 3 , the cells were fixed with Fixation Buffer and permeabilized with Permeabilization/Wash Buffer I (BioLegend). Either (A) unstimulated or (B) stimulated cells were incubated with active caspase- 3 antibody (BD Bioscience) for $20 \mathrm{~min}$ at $4{ }^{\circ} \mathrm{C}$. Finally, cells were washed and analyzed with Attune flow cytometry.

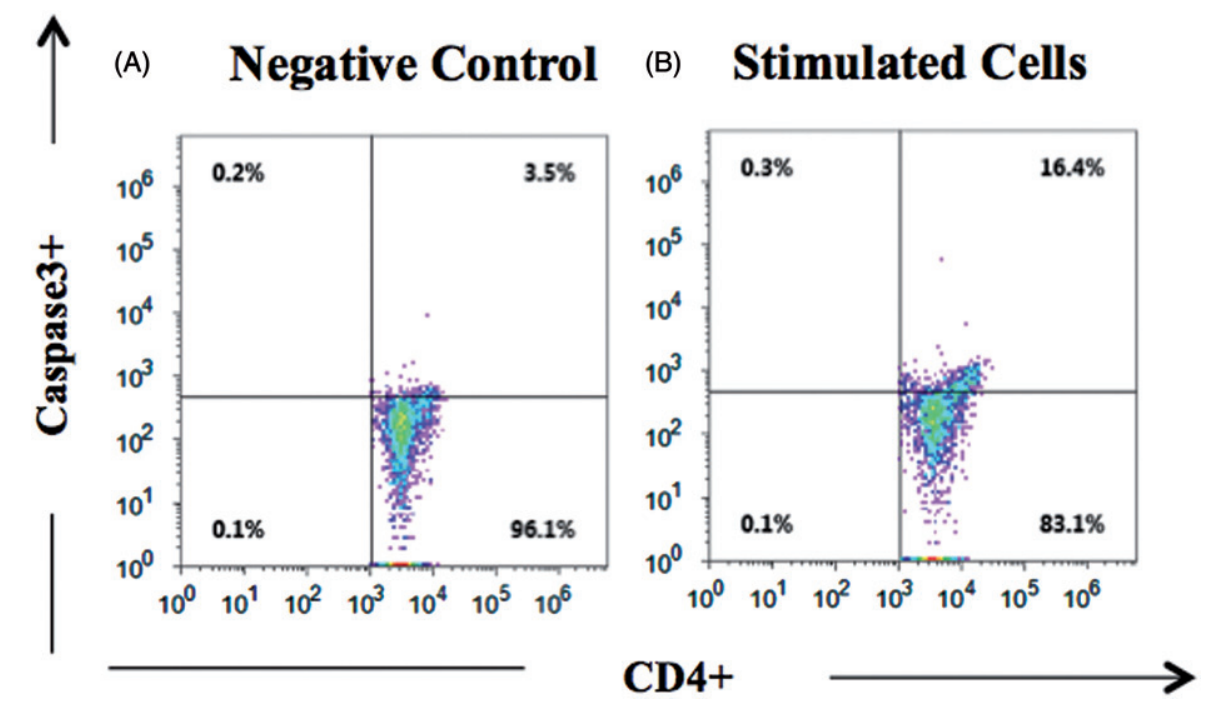

(B)

7he cell viability measurement by 7AAD. Naive CD4 + T cells were stimulated with Th17 polarizing conditions for $7 \mathrm{~d}$. Stimulated naïve CD4 + T cells were labeled with 7AAD and CD4. The cells gated on CD4 positive cells. The viability of CD4 T cells were measured by 7-AAD staining protocol (BD Biosciences). The distinction between positive and negative is clear. Rectangular gates indicate $7 \mathrm{AAD}$ positive cells in naïve $\mathrm{CD} 4+\mathrm{T}$ cell population.
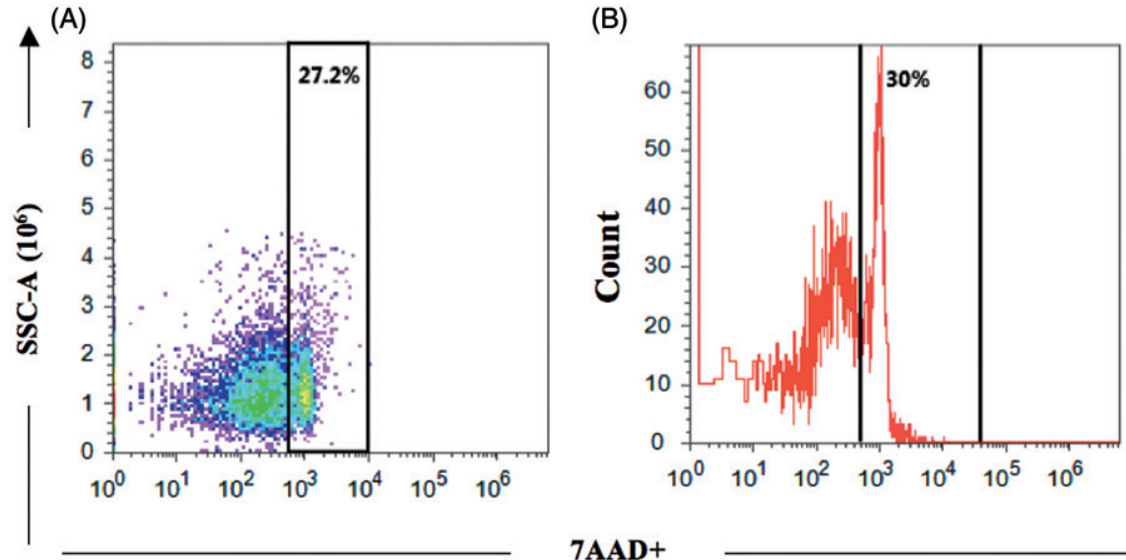

7AAD+ quantification of apoptosis (programmed cell death) at the single cell level based on labeling of DNA strand breaks (Wlodkowic et al., 2009). In the TUNEL assay, (Apo-BrDU) exogenous TdT is used to catalyze a template-independent addition of bromodeoxyuridine triphosphates (Br-dUTP) to the free 3'-hydroxyl ends of double or single-stranded DNA fragments. These cells are visualized by flow cytometry.

\section{Intracellular cytokine detection}

Culturing of the cells was carried out as described above except for intracellular cytokine staining. To detect intracellular cytokines, cultured cells were treated with Golgi stop (1:1500) for $4 \mathrm{~h}$ at $37^{\circ} \mathrm{C}$. At the end of incubation, cells were first washed and labeled with cell surface markers for $\mathrm{T}$ cells. Following washing, cells were fixed and permeabilized for intracellular staining of cytokines. After fixation and permeabilization, fluorescence-conjugated antibody for a target molecule was added to the cells. Finally, the labeled cells were analyzed by flow cytometry (Figure 19).

\section{Recent developments in flow cytometry}

One of the most important progresses in flow cytometry technology is the discovery of Quantum dots (QDs) that provide a great opportunity for highly multiplexed experiments and improved resolution. QDs produced from semiconductor materials such as selenium and cadmium are fluorescent nanocrystals $(2-6 \mathrm{~nm}$ in size) with different properties as compared to small and tandem dyes mentioned (Akinfieva et al., 2013). QDs do not have an absorption spectrum unlike FITC and PE; instead, they have a wide absorption spectrum and so can be excited by a range of different wavelengths. They are also extremely photostable at wavelengths greater than $300 \mathrm{~nm}$ and their different colors can be excited simultaneously by the same source (Buranda et al., 2011; Moquin et al., 2015). There are a wide variety of QDs developed by the commercial companies that are named based on the wavelength of their peak emission including QD545, QD605 and QD655 (Chattopadhyay, 2011). Currently, QDs conjugated to protein A or streptavidin or as secondary antibody conjugates are also available. Antibodyconjugated QDs are suitable to stain both surface antigens and intracellular antigens (Buranda et al., 2011). QDs have been used in a broad range of applications due to their superior properties including the labeling of several targets (cell surface receptor, nuclear antigens) located in different compartments of different samples (tissue samples, cultured live cells and fixed cells) and detection of multiple cancer markers 
(A)

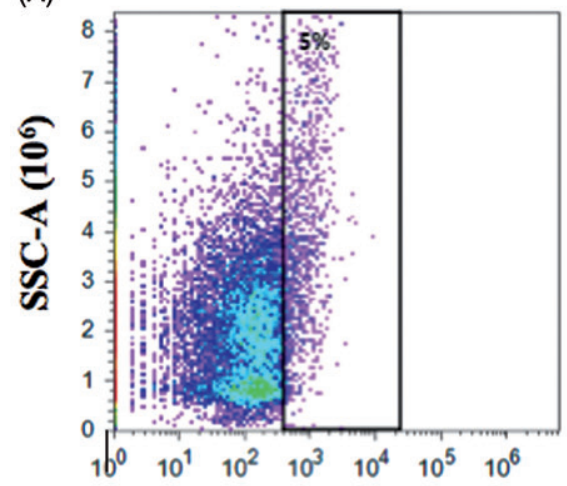

(B)

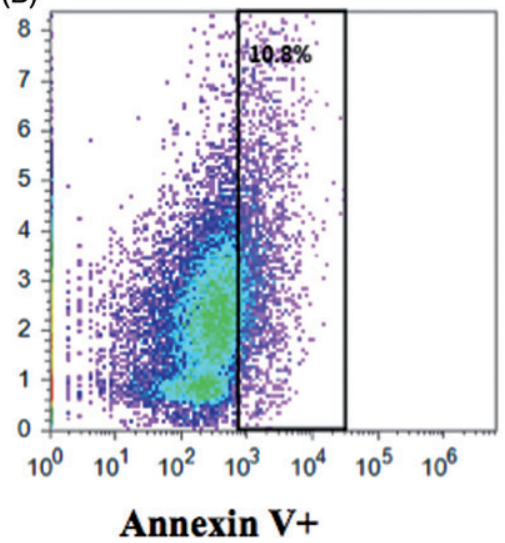

(C)

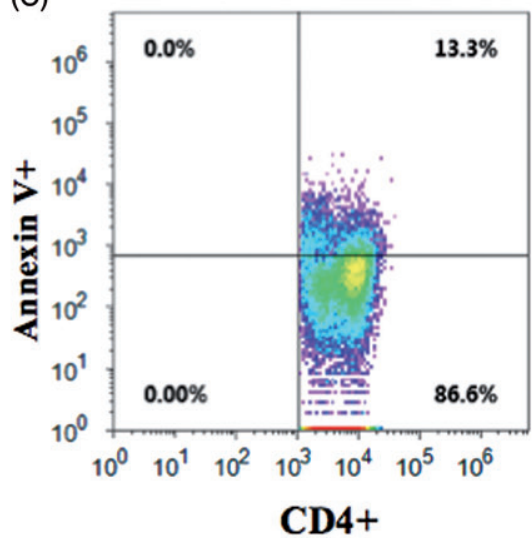

7AAD+

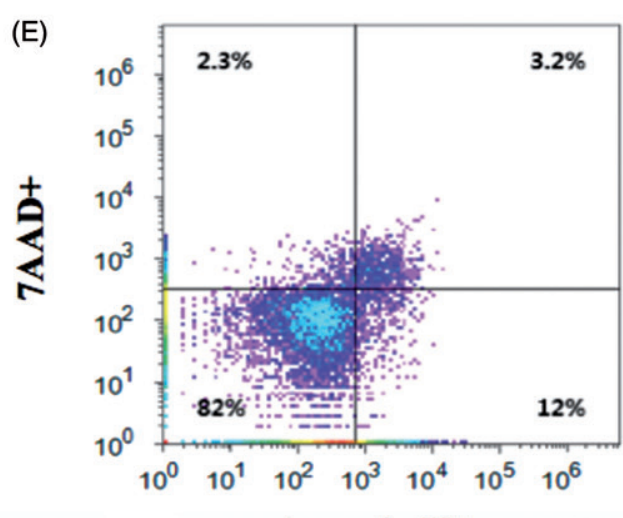

CD4+

Annexin V+

Figure 18. Quantification of apoptosis in CD4 positive T cells with Annexin V/7AAD staining. Naive CD4 + T cells were stimulated with Th17 culture conditions during $7 \mathrm{~d}$. Stimulated naïve CD4 + T cells were labeled with 7AAD, Annexin V and CD4. The cells gated on CD4 positive cells. Viability of CD4 T cells was measured according to 7-AAD and Annexin V staining protocol (BD Biosciences). (A,B) Representative side scatters (SSC) versus 7-AAD and Annexin V density plot. (C,D) Upper right and lower left quadrant shows Annexin V/CD4 and 7AAD/CD4 double positive cells. (E) Cells in the lower right quadrant indicate Annexin-positive/7AAD negative, meaning early apoptotic cells while the cells in the upper right quadrant indicate Annexin-positive/7AAD positive, late apoptotic cells.

(Akinfieva et al., 2013). QDs are also considered as more efficient FRET donors as compared to conventional fluorophores, thus, they are used to study intracellular interactions and binding events (Akinfieva et al., 2013).

In recent years, the development of spectral flow cytometers has enabled fast and sensitive high-resolution measurements of the cell and other particles compared with conventional flow cytometry. The great efforts accomplished by spectral flow cytometers are related to their unique instrumental design, which is different from conventional flow cytometers in terms of optics and detectors (Nolan \& Condello, 2013). Optics (dichroic mirrors) in conventional flow cytometry transmits, block or reflect a photon based on its wavelength while photons are dispersed in a spectral flow cytometer by using prisms and gratings as dispersive optics. A linear array of detectors is commonly used in spectral flow cytometry to measure the continuous spectrum produced by the prism or grating. Currently, charge coupled devices and multianode PMTs are the detectors used in spectral flow cytometers (Nolan et al., 2013). The biological applications of spectral flow cytometry are still under investigation and most of the work with this technique are related to validation studies. For instance, in a recent study, spectral flow cytometry has been used to analyze five different engineered bacterial fluorescent proteins without any spectral overlap (Telford et al., 2015).
In the field of flow cytometry, many advantages have been provided by the development of microfabricated flow cytometers that use microfluidics. Microfluidics are defined as cutting-edge science and technology in which small amounts of fluids $\left(10^{-9}-10^{-18}\right.$ liters $)$ can be processed and manipulated in channels with very small dimensions (Chen et al., 2015). Therefore, microfluidic flow cytometers can be used to analyze single cells in a small population, cellular differences in gene expression or response to a drug within a population of cells (Cheung et al., 2010). These chip-based flow cytometers are cost- and size-effective and portable when compared to conventional benchtop instruments (Cheung et al., 2010). Microfluidic flow cytometers have several advantages over conventional flow cytometers. For instance, a typical flow cytometer delivers a sample of interest at rates of $10-100 \mu \mathrm{l}$ per minute, which is not practical to detect the cells such as circulating tumor cells and hematopoietic stem cells found in very small numbers in the total sample. Because, conventional flow cytometers require analysis of several milliliters of the sample to detect small numbers of cells. However, microfluidic flow cytometers have the ability to concentrate cells and particles at the center of a microfluidic channel for direct analysis, which is based on acoustic concentration (Piyasena \& Graves, 2014). Another advantage of microfluidic flow cytometers is 
Figure 19. Expression of IL17 cytokine. Th17 cultures were terminated according to the expression level of IL-17 (7th or 9th d) and cells were stimulated by $10 \mathrm{ng} / \mathrm{ml}$ IL-23 for every $5 \mathrm{~d}$ in order to refresh the culture condition. Cells are restimulated for $4 \mathrm{~h}$ with Golgi Stop (BD Bioscience). The cells were fixed with Fixation Buffer and permeabilized with Permeabilization/Wash Buffer I

(BioLegend). Both unstimulated and stimulated cells were stained with Anti-Human IL17A Fluorescein-conjugated Antibody

(BioLegend). Finally, cells were analyzed by Attune flow Cytometer and populations were all CD4 gated. Naive CD4 + T cells were gated using FSC/SSC parameters in (A) unstimulated and (B) stimulated Th17 cells. Rectangular gate indicates CD4 and IL17 positive in $(\mathrm{C}, \mathrm{E})$ unstimulated negative control and (D,F) stimulated cells. Cells in the lower left and right quadrant show IL17positive/CD4 positive cells in (G) unstimulated negative control (Figure 19G) and $(\mathrm{H})$ stimulated Th17 cells.

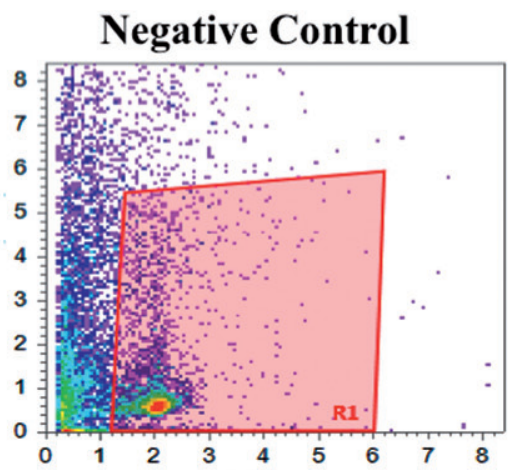

(A)

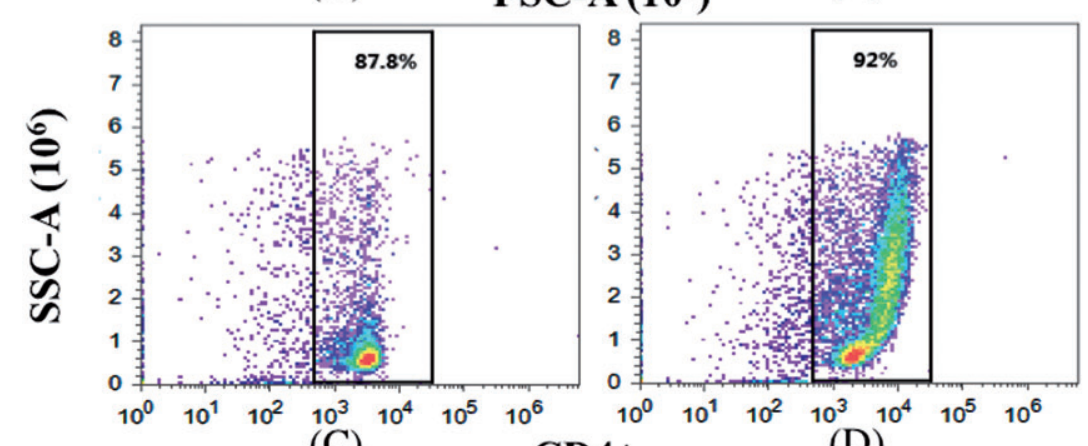

(C) $\mathrm{CD} 4+$

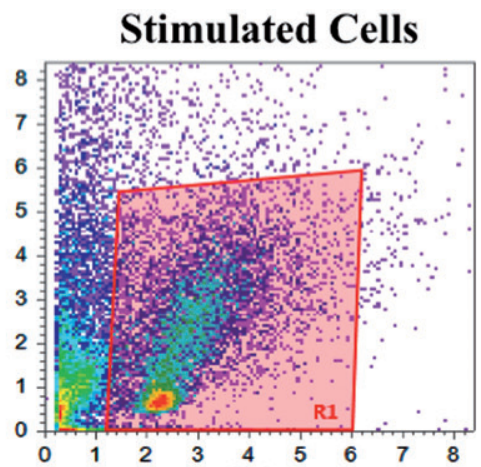

(B)

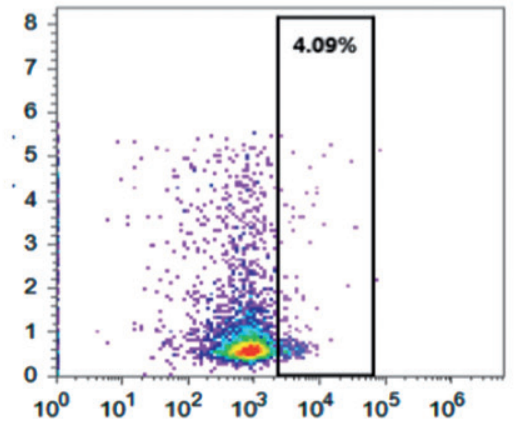

(E)

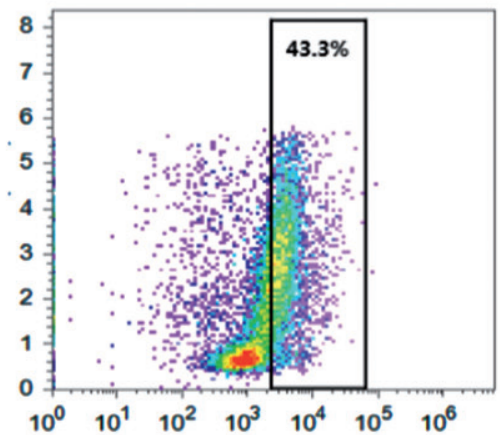

(F)

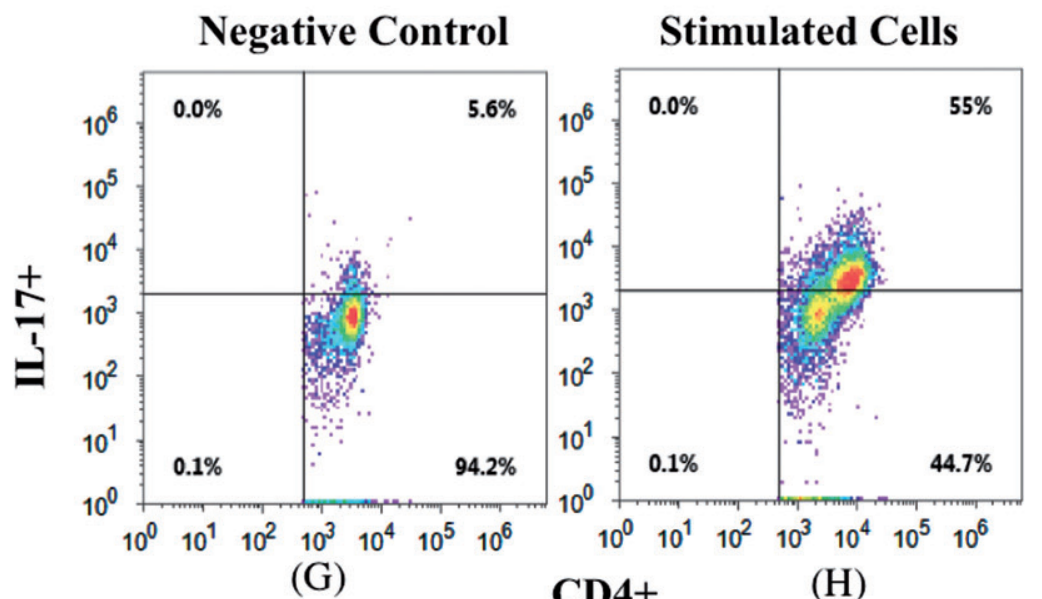

(G) CD4+ high-throughput screening in which small volumes of hundreds of thousands of samples can be analyzed simultaneously. This approach has been used to analyze the cells for high protein content screening in 384 microfluidic flow channels, which uses laser scanning to analyze samples in parallel (McKenna et al., 2011).

\section{Conclusion}

Flow cytometry is one of the most powerful approaches for the analysis of various kinds of samples in a short period of time, which gives valuable information about the question of interest. In this review, the working principle of flow 
cytometry has been emphasized in detail and its common applications, by using data gathered from our own studies, have been discussed to provide a general overview for data generation and analysis. Although new developments have been achieved in the flow cytometry area over the last century, it has continued to adopt innovations in order to reduce cost, size and complexity and to increase sensitivity. These innovations include the development of more efficient fluorochromes such as QDs and of high technology flow cytometers including spectral and microfluidic flow cytometers as discussed throughout the text. It is clear that efforts will continue to optimize and develop flow cytometry, which will ultimately result in significant improvements in several areas such as basic science, medical and biomedical researches.

\section{Declaration of interest}

This work was supported by a grant from the Scientific and Technological Research Council of Turkey (TUBITAK) (Project\# 110T412 to Dr. Ayten Nalbant). The funders had no role in study design, data collection and analysis, decision to publish, or preparation of the manuscript. The authors do not have any kind of conflict of interest affecting the compilation of the current knowledge in this area for writing this review. They apologize to the ones whose elegant studies are not included here because of space limitations.

\section{References}

Akinfieva O, Nabiev I, Sukhanova A. (2013). New directions in quantum dot-based cytometry detection of cancer serum markers and tumor cells. Crit Rev Oncol Hematol, 86, 1-14.

Böyum A. (1968). Isolation of mononuclear cells and granulocytes from human blood. Isolation of mononuclear cells by one centrifugation, and of granulocytes by combining centrifugation and sedimentation at 1 g. Scand J Clin Lab Invest Suppl, 97, 77-89.

Buranda T, Wu Y, Sklar LA. (2011). Quantum dots for quantitative flow cytometry. Methods Mol Biol, 699, 67-84.

Chattopadhyay PK. (2011). Quantum dot technology in flow cytometry. Methods Cell Biol, 102, 463-77.

Chen J, Xue C, Zhao Y, et al. (2015). Microfluidic impedance flow cytometry enabling high-throughput single-cell electrical property characterization. Int J Mol Sci, 16, 9804-30.

Cheung KC, Di Berardino M, Schade-Kampmann G, et al. (2010). Microfluidic impedance-based flow cytometry. Cytometry A, 77, 648-66.

Davies D. (2010). Cell sorting by flow cytometry. In: Macey MG, ed. Flow cytometry: principles and applications. Totowa (NJ): Humana Press, 257-76.

Hulspas R, Dombkowski D, Preffer F, et al. (2009). Flow cytometry and the stability of phycoerythrin-tandem dye conjugates. Cytometry A, $75,966-72$.

Leavesley SJ, Britain AL, Cichon LK, et al. (2013). Assessing FRET using spectral techniques. Cytometry A, 83, 898-912.
Lessene G, Czabotar PE, Colman PM. (2008). BCL-2 family antagonists for cancer therapy. Nat Rev Drug Discov, 7, 989-1000.

Macey MG. (2010). Principles of flow cytometry. Flow cytometry: principles and applications In: Macey MG, ed. Totowa (NJ): Humana Press, 1-15.

McKenna BK, Evans JG, Cheung MC, Ehrlich DJ. (2011). A parallel microfluidic flow cytometer for high-content screening. Nat Methods, 8, 401-3.

Moquin A, Neibert KD, Maysinger D, Winnik FM. (2015). Quantum dot agglomerates in biological media and their characterization by asymmetrical flow field-flow fractionation. Eur J Pharm Biopharm, 89, 290-9.

Nolan JP, Condello D, Duggan E, et al. (2013). Visible and near infrared fluorescence spectral flow cytometry. Cytometry A, 83, 253-64.

Nolan JP, Condello D. (2013). Spectral flow cytometry. Curr Protoc Cytom, Chapter 1:Unit1.27.

Ormerod MG. (2009). Flow cytometry: A basic introduction. Chapter 3: Fluorescence and fluorochromes. ISBN 978-0-9559812-0-3.

Piyasena ME, Graves SW. (2014). The intersection of flow cytometry with microfluidics and microfabrication. Lab Chip, 14, 1044-59.

Reggeti F, Bienzle D. (2011). Flow cytometry in veterinary oncology. Vet Pathol, 48, 223-35.

Roederer M. (2001). Spectral compensation for flow cytometry: visualization artifacts, limitations, and caveats. Cytometry, 45, 194-205.

Shapiro HM. (2004). Lasers for flow cytometry. Curr Protoc Cytom, 49, 1.1.9-.1.17.

Snow C. (2004). Flow cytometer electronics. Cytometry A, 57, 63-9.

Telford WG. (2015). Near infrared lasers in flow cytometry. Methods. doi: 10.1016/j.ymeth.2015.03.010. Epub 2015 Mar 24.

Telford WG, Hawley T, Subach F, et al. (2012). Flow cytometry of fluorescent proteins. Methods, 57, 318-30.

Telford WG, Komoriya A, Packard BZ, Bagwell CB. (2011). Multiparametric analysis of apoptosis by flow cytometry. Methods Mol Biol, 699, 203-27.

Telford WG, Shcherbakova DM, Buschke D, et al. (2015). Multiparametric flow cytometry using near-infrared fluorescent proteins engineered from bacterial phytochromes. PLoS One, 10, $\mathrm{e} 0122342$.

Vermes I, Haanen C, Steffens-Nakken H, Reutelingsperger C. (1995). A novel assay for apoptosis. Flow cytometric detection of phosphatidylserine expression on early apoptotic cells using fluorescein labelled Annexin V. J Immunol Methods, 184, 39-51.

Wilkerson MJ. (2012). Principles and applications of flow cytometry and cell sorting in companion animal medicine. Vet Clin North Am Small Anim Pract, 42, 53-71.

Wlodkowic D, Skommer J, Akagi J, et al. (2013). Multiparameter analysis of apoptosis using lab-on-a-chip flow cytometry. Curr Protoc Cytom, 66, 9.42.1-.42.15

Wlodkowic D, Skommer J, Darzynkiewicz Z. (2009). Flow cytometrybased apoptosis detection. Methods Mol Biol, 559, 19-32.

Wlodkowic D, Skommer J, Darzynkiewicz Z. (2011a). Rapid quantification of cell viability and apoptosis in B-cell lymphoma cultures using cyanine SYTO probes. Methods Mol Biol, 740, 81-9.

Wlodkowic D, Skommer J, Darzynkiewicz Z. (2012). Cytometry of apoptosis. Historical perspective and new advances. Exp Oncol, 34, 255-62.

Wlodkowic D, Telford W, Skommer J, Darzynkiewicz Z. (2011b). Apoptosis and beyond: cytometry in studies of programmed cell death. Methods Cell Biol, 103, 55-98. 